\title{
Dinâmica populacional de Aegla platensis Schmitt (Crustacea, Decapoda, Aeglidae) $^{1}$
}

\author{
Alessandra Angélica de Pádua Bueno ${ }^{2}$ \\ Georgina Bond-Buckup ${ }^{2}$
}

\begin{abstract}
Population's dynamics of Aegla platensis Schmitt (Crustacea, Decapoda, Aeglidae). The crustaceans of the genus Aegla Leach, 1820, are found in streams, rivers of caves, current rivers and lagoons, from Franca, São Paulo, Brazil $\left(20^{\circ} 60^{\prime} \mathrm{S}, 47^{\circ} 40^{\prime} \mathrm{W}\right)$, to Ilha Madre de Dios ( $\left.50^{\circ} 01^{\prime} 10^{\prime \prime} \mathrm{S}, 75^{\circ} 18^{\prime} 45^{\prime \prime} \mathrm{W}\right)$, Chile in South America. The crabs (Aegla platensis Schmitt, 1942) were monthly sampled by hand from july 1997 to september 1998 in Arroio do Mineiro, Taquara (2946'S, 5053'W), Rio Grande do Sul, Brazil. The sex, the length and width of the carapace were registered. After being collected the animals were returned to the stream. The reproductive period, the size at the first maturation and the sex-ratio were identified. The reproductive period of $A$. platensis lasts the whole year. The percentage of ovigerous females is higher in july. The size at the first maturation is from 14,40 to $15,60 \mathrm{~mm}$ of carapace length. The sex-ratio is 1,08 male to 1 female. The annual population's density is 12 animals per square meter.

KEY WORDS. Crustacea, Aegla platensis, reproduction and population dynamics
\end{abstract}

Os aeglídeos são um grupo de crustáceos peculiares por serem os únicos anomuros de águas continentais, sendo encontrados somente na América do Sul, ocorrem em arroios, rios de correnteza e lagos desde o município de Franca, São Paulo, Brasil, até a ilha Madre de Dios, no Chile (BOND-BUCKUP \& BUCKUP 1994). Fazem parte da cadeia alimentar dos ambientes límnicos, sendo considerados importantes na dieta de rãs, peixes e aves (ARENAS 1974), alimentando-se de larvas de insetos simulídeos (MAGNI \& PY-DANIEL 1989).

Aspectos da reprodução e do crescimento de Aegla laevis laveis (Latreille, 1818) foram abordados por BAHAMONDE \& LOPEZ (1961) no Chile, onde verificaram o período reprodutivo, o tamanho da primeira maturação e a proporção de machos por fềmeas. No Brasil, LOPEZ (1965) estudou a reprodução de Aegla odebrechtti paulensis Schmitt, 1942 (hoje A. paulensis) em São Paulo. JARA (1977) fez algumas observações sobre a biologia de Aegla rostrata Jara, 1977, no Chile. RODRIGUES \& HEBLING (1978) estudaram aspectos biológicos e biométricos de Aegla perobae Hebling \& Rodrigues, 1977, em São Paulo.

Diante dos escassos trabalhos sobre a dinâmica populacional do grupo, o presente estudo trata dos aspectos bio-ecológicos de Aegla platensis Schmitt, 1942, em especial o período reprodutivo e o tamanho da população em ambiente natural.

1) Contribuição número 305 do Departamento de Zoologia, Instituto de Biociências, Universidade Federal do Rio Grande do Sul.

2) Programa de Pós-Graduação em Biologia Animal, Departamento de Zoologia Instituto de Biociências, Universidade Federal do Rio Grande do Sul. Avenida Paulo Gama, prédio 12105, 90040-060 Porto Alegre, Rio Grande do Sul, Brasil. 


\section{MATERIAL E MÉTODOS}

Exemplares de Aegla platensis foram coletados de julho/97 a setembro/98, no Arroio do Mineiro, localidade de Fazenda Fialho, município de Taquara $\left(29^{\circ} 46^{\prime} \mathrm{S}\right.$ $\left.-50^{\circ} 53^{\prime} \mathrm{W}\right)$, Rio Grande do Sul. Com os resultados de coletas pilotos, verificou-se que em determinado local do arroio os animais eram mais abundantes, optando-se, portanto, por coletar os animais neste trecho com extensão aproximada de $18 \mathrm{~m}$.

Em cada saída à campo, os animais foram capturados manualmente com auxílio de vários puçás. A coleta foi exaustiva, levantando-se todas as pedras e mexendo-se no substrato e na vegetação de uma margem a outra do arroio, sempre no sentido oposto da correnteza, para que tanto jovens e adultos pudessem cair nos puçás.

Foram registrados, para cada exemplar, o sexo, o comprimento e a largura do cefalotórax, assim como, a presença de fêmeas ovígeras. Para as medidas, utilizou-se um paquímetro digital com precisão de $0,1 \mathrm{~mm}$. Em cada coleta foram medidos o $\mathrm{pH}$ e a temperatura da água em diferentes pontos do arroio, utilizando um medidor de $\mathrm{pH}$ portátil e termômetros com escala interna.

$\mathrm{Na}$ estimativa do tamanho da população e da observação do deslocamento dos animais no arroio utilizou-se a estimativa de Petersen, cujo modelo encontra-se descrito em BEGON (1979). Considerou-se, ainda, as premissas de captura-recaptura indicadas para o modelo. Os animais coletados sazonalmente foram marcados com um ponto, com uma caneta marcadora de cor prateada, no cefalotórax. Depois da marcação de todos os exemplares, os animais foram devolvidos ao arroio no local em que foram coletados. Após dois dias, tempo suficiente para que os animais marcados se misturassem aos demais na população e para que as marcas não fossem perdidas, os indivíduos foram coletados novamente e observados os marcados e não marcados. Em cada extremo do arroio (após os $18 \mathrm{~m}$ ) coletou-se de metro em metro até não encontrar mais nenhum animal marcado.

\section{Análise estatística}

O periodo reprodutivo foi verificado através da presença de fêmeas ovígeras ao longo do ano. Estimou-se o comprimento médio do início da primeira maturação sexual (a partir do momento em que $50 \%$ das fêmeas constituintes da população estavam ovígeras) (VAZZOLER 1981).

Para verificar a possível ocorrência de diferenças significativas na proporção de machos e fêmeas ao longo do ano, foi utilizado o teste $\mathrm{X}^{2}$ (qui-quadrado), adotando-se as recomendações de SNEDERCOR \& COCHRAN (1967). O X ${ }^{2}$ calculado foi comparado com o valor do $\mathrm{X}^{2}$ crítico a nível de $5 \%$ de significância e $\mathrm{n}-1$ graus de liberdade $(n=2)$.

Para a estimativa do tamanho da população, através do método de marcação e recaptura, foi utilizada a estimativa de Petersen: $\mathbf{N}=\mathbf{r} . \mathbf{n} / \mathbf{m}$, onde: $(\mathrm{N})$ estimativa do tamanho populacional; (r) número de animais marcados no primeiro dia; (n) número de animais coletados no segundo dia; $(\mathrm{m})$ o número de animais recapturados com marcas.

Os resultados da estimativa da população foram transformados em densida$\mathrm{de} / \mathrm{m}^{2}$ média anual e por estação do ano, dividindo-se os valores por $90 \mathrm{~m}^{2}$, correspondente a área do arroio amostrada. 


\section{RESULTADOS E DISCUSSÃO}

O período reprodutivo observado de Aegla platensis estende-se por todo ano, embora nos meses de novembro e dezembro não terem sido coletadas fêmeas ovígeras. No entanto, em coletas pilotos realizadas nos meses de outubro, novembro e dezembro de 1996, verificou-se a presença de fêmeas ovígeras.

Em espécies chilenas de Aegla o período reprodutivo não ultrapassa oito meses, como foi observado por BAHAMONDE \& LOPEZ (1961) em A. laevis laevis onde este período foi de março a outubro.

SASTRY (1983) analisando os fatores que interferem no período reprodutivo, destaca que este pode estar sincronizado com vários fatores ambientais, como a duração do dia, a temperatura e mudanças na salinidade, no caso de crustáceos marinhos. A interação destes fatores e as condições fisiológicas favoráveis dos crustáceos podem prolongar o período reprodutivo. O prolongado período de reprodução de $A$. platensis pode estar relacionado a abundância de recursos alimentares, temperaturas amenas $\left(13^{\circ} \mathrm{C}\right.$ no inverno e $22^{\circ} \mathrm{C}$ no verão) e águas límpidas.

$\mathrm{O}$ maior número de fêmeas ovígeras de $A$. platensis ocorreu no mês de julho onde $20 \%$ das fêmeas coletadas estavam ovígeras (Fig. 1). Em A. paulensis, LOPEZ (1965) também verificou que a maior freqüência de fêmeas ovígeras ocorreu durante o inverno (junho) e o surgimento dos juvenis na população no início da primavera (setembro). JARA (1977) registrou a presença de fêmeas ovígeras de Aegla rostrata somente no mês de abril.

Através da curva de crescimento, estimou-se o tamanho da primeira maturação entre 14,40 e 15,60 mm de comprimento do cefalotórax, ocorrendo quando a fêmea está entre 378 e 467 dias de vida (BUENo et al. 2000). Porém, a menor e a maior fêmea ovígera apresentaram 9,87 e 17,72 mm de comprimento do cefalotórax, respectivamente (Fig. 2). BAHAMONDE \& LOPEZ (1961) verificaram que a primeira maturação sexual de $A$. laevis laevis, espécie chilena, ocorre quando a fêmea apresenta $12,5 \mathrm{~mm}$ de comprimento do cefalotórax.

A razão sexual média obtida para $A$. platensis foi de 1,08 machos para cada fêmea (Fig. 3). Ao longo do ano não houve diferenças significativas na proporção de machos e fêmeas na população (Tab. I). Estes resultados corroboram as observações de BAHAMONDE \& LOPEZ (1961) para A. laevis laevis onde a proporção macho/ fêmea foi de 1:1, com exceção dos meses de junho e dezembro onde esta razão foi de 1:5. LOPEZ (1965) também verificou a mesma proporção (1:1) para $A$. paulensis. Já RODRIGUES \& HEBLING (1978) encontraram uma proporção bem maior de machos de $A$. perobae (7:3 e $5: 1$ ).

Em observações feitas no campo, os exemplares juvenis e adultos de Aegla platensis mostraram preferências por determinados locais. Em muitas observações, constatou-se que as fềmeas ovígeras escolhem a margem próxima à vegetação. Os juvenis, por sua vez, preferem os locais com pouca correnteza e substrato de areia mais fina.

Examinando os dados relativos ao crescimento de $A$. platensis, e considerando os artefatos de coleta utilizados, verifica-se o surgimento dos juvenis em agosto (BUENo et al. 2000). Através da observação da idade e do tamanho da primeira maturação (14,40 mm e 378 dias), constata-se que os juvenis se reproduzem 

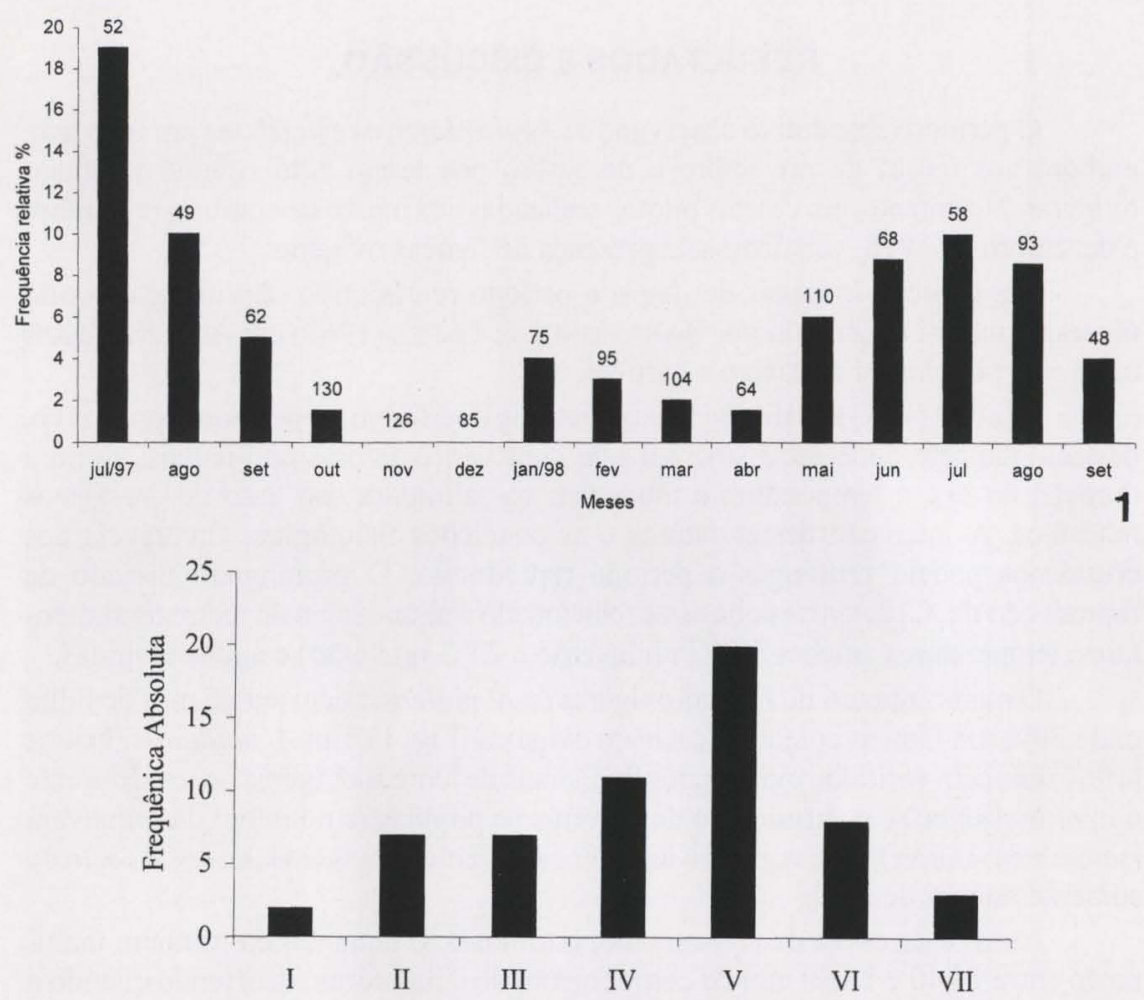

Classes de Comprimento do Cefalotórax
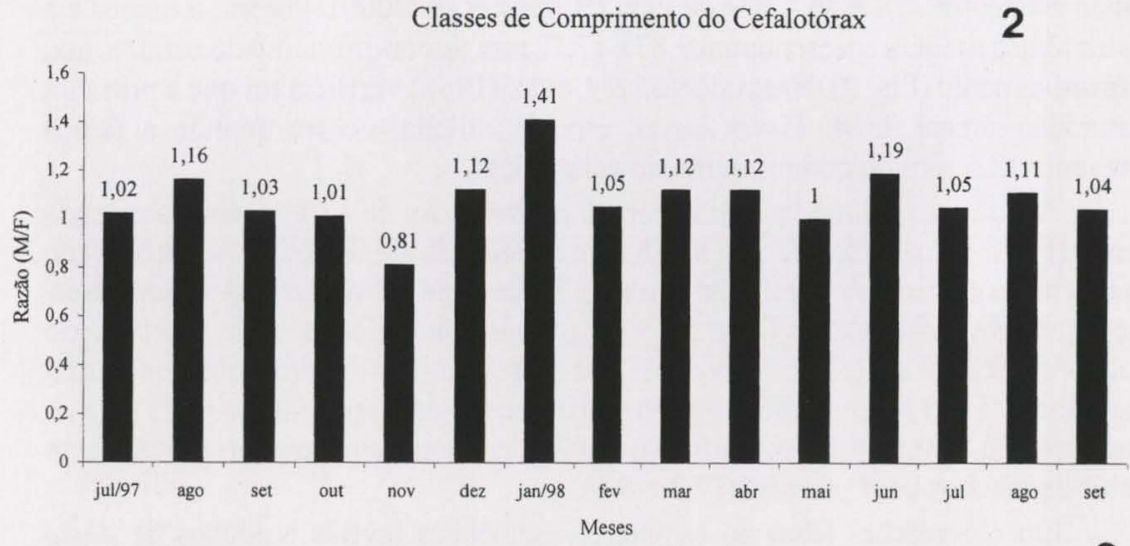

Figs 1-3. Aegla platensis, amostrada de julho/97 a setembro/98, no Arroio do Mineiro, município de Taquara, Rio Grande do Sul. (1) Freqüências relativas de fêmeas ovígeras, por mês de coleta; (2) Freqüências absolutas de fêmeas ovigeras por intervalo de classes de comprimento do cefalotórax (mm) (I: $9,87 \mid-10,8$; II: 10,8|- 12,0; III: 12, 이-13,2; IV: 13,2 |-14,4; V: 14,4 |- 15,6; VI: 15,6 |- 16,8; VII: 16,8 |-| 18,0); (3) Razões macho/fêmea por mês de amostragem. Na figura 1 o número acima de cada coluna de amostragem é o total de fêmeas coletadas. 
após completar um ano. Estes passarão por mais um ano de vida, chegando pelo menos a dois anos e meio (Fig. 4). Estima-se este resultado através da curva de crescimento, onde os animais maiores atingem $17,39 \mathrm{~mm}$ de comprimento do cefalotórax (machos) e 19,12mm (fêmeas).

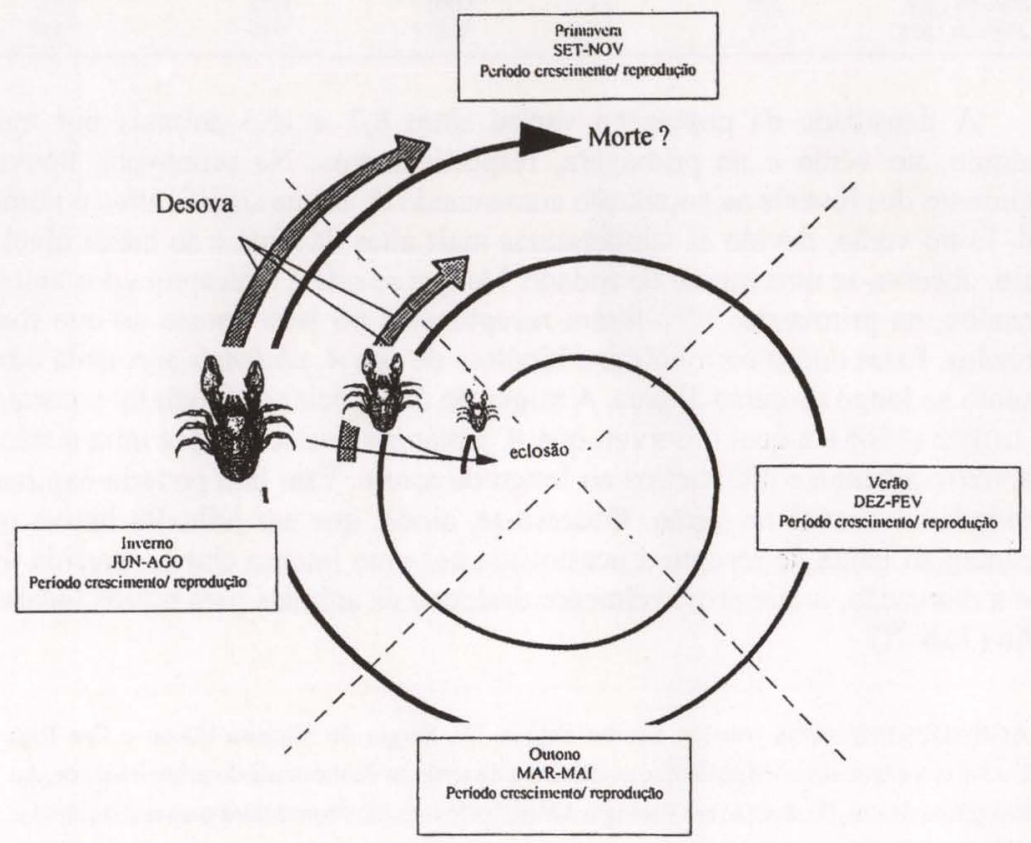

Fig. 4. Hipótese da história de vida de Aegla platensis, no Arroio do Mineiro, município de Taquara, Rio Grande do Sul.

Tabela I. Aegla platensis. Freqüências absolutas e o teste do $X^{2}$ de machos e fêmeas de indivíduos capturados de julho/97 a setembro/98, no Arroio do Mineiro, município de Taquara, Rio Grande do Sul.

\begin{tabular}{lcccc}
\hline \multicolumn{1}{c}{ Meses } & Machos & Fèmeas & Machos/fêmeas & $X^{2}$ \\
\hline Julho de 1997 & 53 & 52 & 1,02 & $0,0095 \mathrm{NS}$ \\
Agosto & 57 & 49 & 1,16 & $0,6036 \mathrm{NS}$ \\
Setembro & 64 & 62 & 1,03 & $0,0316 \mathrm{NS}$ \\
Outubro & 132 & 130 & 1,01 & $0,0152 \mathrm{NS}$ \\
Novembro & 102 & 126 & 0,81 & $2,5262 \mathrm{NS}$ \\
Dazembro & 95 & 75 & 1,12 & 0,5554 NS \\
Janeiro de 1998 & 106 & 95 & 1,41 & $5,3148{ }^{*}$ \\
Fevereiro & 100 & 104 & 1,05 & $0,1282 \mathrm{NS}$ \\
Março & 117 & 110 & 1,12 & $0,7646 \mathrm{NS}$ \\
Abril & 72 & 68 & 1,12 & $0,4706 \mathrm{NS}$ \\
Maio & 110 & 58 & 1,00 & $0,0000 \mathrm{NS}$ \\
Junho & 81 & 93 & 1,19 & $1,1342 \mathrm{NS}$ \\
Julho & 61 & 48 & 1,05 & $0,0756 \mathrm{NS}$ \\
Agosto & 103 & 1,11 & 0,5102 NS \\
Setembro & 50 & 1,04 & $0,0408 \mathrm{NS}$ \\
\hline
\end{tabular}

$\left({ }^{*}\right)$ significante para $\alpha$ igual ou menor do que 0,05 e maior do que 0,01 ; (NS) não significante para $\alpha$ igual a 0,05 . 
Tabela II. Aegla platensis. Dados quantitativos da estimativa de Petersen do tamanho da população, no Arroio do Mineiro, município de Taquara, Rio Grande do Sul.

\begin{tabular}{lccccc}
\hline Meses & Marcados & Racapturados & Recaptura (\%) & Populaçăo estimada & Animais $/ \mathrm{m}^{2}$ \\
\hline Novembro de 1997 & 153 & 14 & 16,33 & 790 & 8,7 \\
Fevereiro de 1998 & 155 & 25 & 15,50 & 950 & 15,3 \\
Maio de 1998 & 220 & 24 & 13,60 & 1672 & 18,6 \\
Julho de 1998 & 171 & 30 & 4,70 & 1710 & 19,0 \\
\hline
\end{tabular}

A densidade da população variou entre 8,7 a 15,3 animais por metro quadrado, no verão e na primavera, respectivamente. Na primavera, houve o surgimento dos juvenis na população aumentando de forma significativa o número total. Já no verão, devido às temperaturas mais altas da água e ao baixo nível do arroio, observa-se uma menor densidade. No que se refere à recaptura dos animais marcados, na primavera, $32 \%$ foram recapturados no lado oposto ao que foram marcados. Estes dados corroboram a hipótese de que $A$. platensis apresenta deslocamento ao longo do curso d'água. A migração de espécies de Aegla foi constatada por LOPEZ (1965), a qual observou que $A$. paulensis desloca-se por uma distância de aproximadamente 300 metros ao longo do arroio. Este fato poderia explicar a densidade $/ \mathrm{m}^{2}$ menor no verão. Observa-se, ainda, que em julho/98 houve uma percentagem baixa de recaptura ocasionada por uma intensa chuva ocorrida logo após a marcação, o que provavelmente deslocou os animais para outros locais do arroio (Tab. II).

AGRADECIMENTOS. Ao Dr. Sandro Santos, Dr. Sérgio de Siqueira Bueno e Dra Inga Ludmila Veitenheimer-Mendes que avaliaram a dissertação de mestrado do primeiro autor. Ao Programa de Pós-Graduação em Biologia Animal pelos auxílios concedidos para realização dos trabalhos de campo.

\section{REFERÊNCIAS BIBLIOGRÁFICAS}

ARENAS, J. 1974. La cordillera de la costa como refugio de la fauna dulcícola preglacial. Arch. Biol. Med. Exp. 10: 1-40.

Bahamonde, N. \& M.T. Lopez. 1961. Estudios biologicos en la populacion de Aegla laevis laevis (Latreille) de el Monte (Crustacea, Decapoda, Anomura). Investnes zool. Chil. 7: 19-58.

BEGON, M. 1979. Investigating animal abundance: capture-recapture for biologist. London, Edward Arnold, 97p.

Bond-Buckup, G. \& L. Buckup. 1994. A família Aeglidae (Crustacea, Decapoda, Anomura). Arch. Zool. Est. São Paulo 2 (4): 159-346.

Bueno, A.A.P.; G. Bond-BuckuP \& L. Buckup. 2000. Crescimento de Aegla platensis Schmitt, em ambiente natural (Crustacea, Decapoda, Aeglidae). Revta bras. Zool. 17 (1): 51-60.

JARA, C. 1977. Aegla rostrata n.sp., (Decapoda, Aeglidae), nuevo crustáceo dulceacuícola del Sur de Chile. Stud. Neotrop. Fauna Environ. 12: 165-176.

Lopez, M.T. 1965. Estudios biologicos en Aegla odebrechtti paulensis, Schmitt (Crustacea, Decapoda, Anomura). Bol. Zool. Fac. Fil. Cien. Letras, São Paulo, 25: 301-314.

Magni, S. T. \& V. Py-Daniel. 1989. Aegla platensis Schmitt,1942 (Decapoda, Anomura) um predador de imaturos de Simullidae (Diptera, Culicomorpha). Revta Saúde Públ., São Paulo, 23 (3): 258-259.

Rodrigues, W. \& N.J. Hebling. 1978. Estudos biológicos em Aegla perobae Hebling \& Rodrigues, 1977 (Decapoda, Anomura). Rev. Brasil. Biol. 38 (2): 383-390. 
SASTRY, A.N. 1983. Ecological aspects of reproduction, p. 179-2170. In: D.E. BuIss (Ed.). The Biology of Crustacea: Envirommental Adaptation. New York, Academic Press, Vol. 8, 383p.

SNedecor, G.W. \& W.G. Cochran. 1967. Statistical Methods. Ames, Iowa State Univ. Press, $6^{\text {th }}$ ed., 593p.

VAZZolER, A.E.A.M. 1981. Manual de métodos para estudos biológicos de populações de peixes: reprodução e crescimento. Brasília, CNPq, Programa Nacional de Zoologia, 108p.

Recebido em 31.III.1998; aceito em 01.II.2000. 